\title{
Photoinduced magnetic linear dichroism in a YIG:Co film
}

\author{
O. V. Miloslavska, Yu. N. Kharchenko, N. F. Kharchenko, and V. G. Yurko \\ B. Verkin Institute for Low Temperature Physics and Engineering \\ of the National Academy of Science of Ukraine, 47 Lenin Ave., Kharkiv 61103, Ukraine \\ E-mail:miloslavskaya@ilt.kharkov.ua
}

A. Stupakiewicz and A. Maziewski

Laboratory of Magnetism, University of Bialystok, 15-424 Bialystok, Poland

Received November 12, 2001

\begin{abstract}
The spectra of linear dichroism induced by magnetic field are observed in a cobalt-doped yttrium iron garnet (YIG:Co) film grown in the (001) plane. The linear dichroism spectra are highly sensitive to the orientation of the magnetic field. The spectrum measured with the magnetic field directed along the crystallographic axis [100] turned out to have a form identical to the spectrum of the «rigid» photoinduced linear dichroism, known from earlier experiments. The similarity of these spectra may be regarded as evidence of the magnetic origin of the major part of the optical anisotropy induced by light with polarization $\mathbf{E} \|[100]$ in the nonmagnetized YIG:Co film.
\end{abstract}

PACS: 75.30.Gw, 75.50.Gg, 78.20.Ls

\section{Introduction}

Yttrium iron garnets $\mathrm{Y}_{3} \mathrm{Fe}_{5} \mathrm{O}_{12}$ (YIG) doped with $\mathrm{Co}$ ions are interesting and promising objects for investigations and applications [1-6]. Substitution of the isotropic $\mathrm{Fe}^{3+}$ ions, which have zero orbital moment, by strongly anisotropic Co ions results in noticeable changes of the magnetic properties of the material, including the magnetic anisotropy, magnetostriction, and the temperature behavior of the magnetization [1,2]. These substances are important as sensitive photomagnetic materials. Illumination of doped YIG films by visible or nearIR light often results in changes of their magnetic and nonmagnetic properties [3-8]. The appearance of light-induced anisotropic properties of the films is caused by the creation of highly anisotropic photosensitive centers when certain ions with valences different from the host ions are implanted in the YIG crystal lattice [3,4]. In films of yttrium iron garnet doped with cobalt (YIG:Co), especially strong photoinduced changes of the magnetic anisotropy and the magnetic hysteresis loops as well as switching of the magnetic domains by light have been revealed and studied by different methods $[3,5,6]$. The photoinduced effects in YIG:Co were stronger than in YIG crystals doped with $\mathrm{Si}$ ions $[4,8]$. In $[5,6]$ the photoinduced polarization-sensitive changes of the domain structure in epitaxial YIG:Co films have been investigated even at room temperature.

The light-induced optical linear dichroism was also observed in this film in [7]. The spectra of linear dichroism induced by polarized light in a nonmagnetized epitaxial YIG:Co film were studied in the visible region. A dichroism spectrum with a prominent structure has been observed after irradiating the film by light with the plane of polarization along the [100] crystallographic axis of the film. No spectral features were observed when the irradiating light was polarized along [110]. The photoinduced dichroism spectrum could be considered as consisting of two components. The component called «soft» dichroism changes its sign when the plane of polarization of the irradiating light is rotated by $90^{\circ}$. The dichroism spectral bands, which 
do not change sign when the polarization axis of inducing light is rotated by $90^{\circ}$, compose the «rigid» component. The bands of the «rigid» LD spectrum could be observed after irradiation by [100]- or [010]-polarized light. The characteristic feature is the revealed memory phenomenon of the «rigid» dichroism: the photoinduced spectrum, which vanishes on heating up to $170 \mathrm{~K}$ through a change of magnetic order, is restored again after cooling back to $28 \mathrm{~K}$ without irradiation. The authors of [7] supposed that the «rigid» dichroism is a result of the photoinduced reorientation of the magnetic moments of the photoactive ions in the YIG:Co film and that the emergence of the photoinduced dichroism may actually be presented as a manifestation of the magnetooptic Voigt effect which is observed when the magnetic moments are oriented in the plane of the film in a definite way.

The aim of the present work was to verify the assumption about the magnetic origin of the changes of photoinduced optical anisotropy [7] by means of experimental studies of the magnetic dichroism spectra in a YIG:Co film in Voigt the geometry $\mathbf{H} \perp \mathbf{k} \| \mathbf{s}$, where $\mathbf{k}$ is the light propagation vector and $\mathbf{s}$ is the normal to the surface of the film.

\section{Experimental results and discussion}

The garnet film of $\mathrm{Y}_{2} \mathrm{CaFe}_{3.9} \mathrm{Co}_{0.1} \mathrm{GeO}_{12}$ (YIG:Co) was grown by the method of liquid-phase epitaxy on a [001]-oriented substrate of $\mathrm{Gd}_{3} \mathrm{Ga}_{5} \mathrm{O}_{12}$. The thickness of the YIG:Co film is $5.8 \mu \mathrm{m}$. The easy axis of YIG:Co changes its orientation with temperature [9]. In the temperature range between $125 \mathrm{~K}$ and $10 \mathrm{~K}$ three magnetic states can exist, with easy axis along [001], [100], and [110], either in stable or metastable form [10], and at the temperature of the present experiment, $T=30 \mathrm{~K}$, the magnetic state with easy axis along [001] can be assumed to be dominant. The measurements of the magnetic linear dichroism spectra of YIG:Co were carried out in the spectral region $500-800 \mathrm{~nm}$. We used a magnetooptical setup which comprised a monochromator, photoelastic modulator, cryogenic equipment, and superconducting solenoids. The spectral dependence of the value $\left(I_{1}-I_{2}\right) /\left(I_{1}+I_{2}\right)$ was measured using the modulation method, $I_{1}$ and $I_{2}$ being the intensities of light with the orthogonal orientations of the plane of polarization. The spectral measurements were performed with the electric vectors of the light oriented parallel to the magnetic field $\mathbf{H}$ for $I_{1}$ and perpendicular to $\mathbf{H}$ for $I_{2}$. The light coming from monochromator had rather low intensity and did not generate any noticeable photoinduced effect. The cryogenic technique allowed us to obtain temperatures as low as $10 \mathrm{~K}$. The in-plane magnetic field was applied perpendicularly to the light propagation vector. The angle between the magnetic field vector and the cubic crystallographic axis [100] of the sample could be varied by rotation of the sample-holder around the normal to the YIG:Co film surface coinciding with crystallographic axis [001].

A series of spectra of linear dichroism (LD) measured at different orientations of the magnetic field has been obtained at temperature near $30 \mathrm{~K}$. The magnetic field was switched on after zero-field cooling (ZFC). The applied magnetic field strength was $\sim 8.8 \mathrm{kOe}$. This value of the in-plane magnetic field was sufficient for homogeneous magnetization of the film. The orientation of the magnetic field was changed by rotating the sample around its [001] axis, that is, normal to the film surface, at the constant temperature. The LD spectra measured with the magnetic field oriented along the [100] and [110] axes and the spectrum at zero magnetic field are shown in Fig. 1. The forms of the spectra are distinctly different for different orientations of magnetic field. The LD spectrum measured with the magnetic field directed along the [100] axis (Fig. 1,a) has a well-defined structure, and the highest value of the magnetic linear dichroism is attained. We have not noticed any diversity in the spectra obtained in the [100]- and [010]-oriented magnetic fields. However, when the magnetic field was directed along an axis of the [110] type, the LD spectrum was quite different and the linear dichroism value was lowered over almost all of the spectral region studied (Fig. 1,b). At zero field,

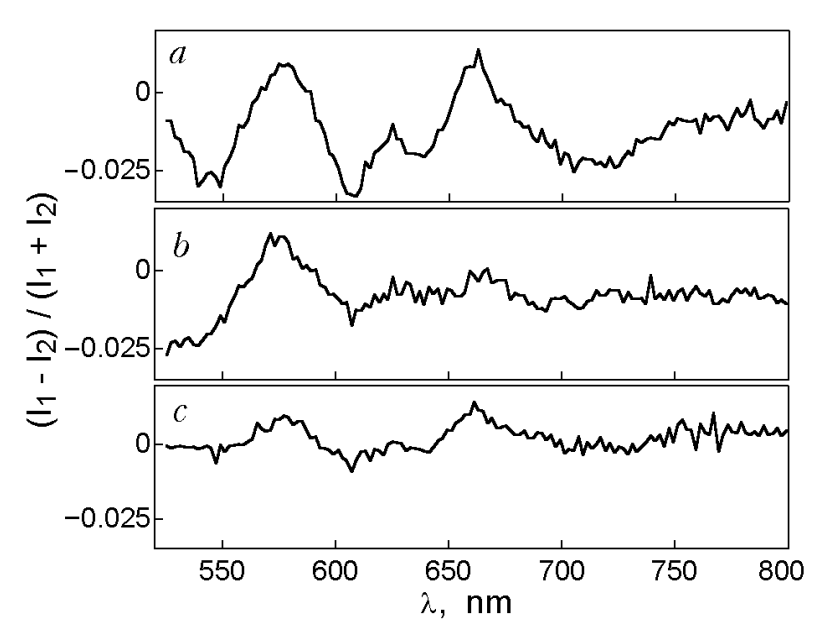

Fig. 1. Magnetic linear dichroism spectra measured after ZFC at $H=8.8 \mathrm{kOe}(T=30 \mathrm{~K})$ for different orientations of the magnetic field: $\mathbf{H}\|[100](a) ; \mathbf{H}\|[110](b)$; and at zero field, $H=0(c)$. 


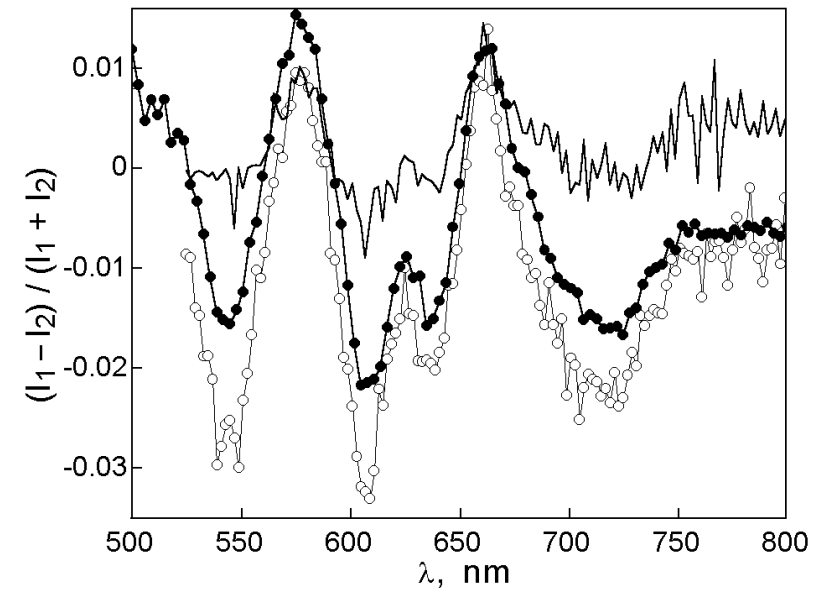

Fig. 2. The spectra of magnetic linear dichroism at $H=8.8$ kOe without irradiation (open circles) at $T=30 \mathrm{~K}$ (the present experiment) and of the photoinduced LD after irradiation with $\mathbf{E} \|[100]$ (solid symbols) at $T=28 \mathrm{~K}$ [7]. The latter has been multiplied by five. The line without symbols shows the $\mathrm{LD}$ at $H=0$ and without irradiation.

when the sample is not magnetically homogeneous, the LD is small (Fig. 1,c).

A comparison of the spectrum of magnetic linear dichroism induced by $\mathbf{H} \|[100]$ with the LD spectrum induced by the action of polarized light with $\mathbf{E} \|$ [100] observed in [7] is shown in Fig. 2. The sample has been irradiated with linearly polarized light coming either from a white light source through a filter with $\lambda>640 \mathrm{~nm}$ or from a heliumneon laser with $\lambda=632 \mathrm{~nm}$. The illumination was carried out for about $20 \mathrm{~min}$, at a power about $0.4 \mathrm{~W} / \mathrm{cm}^{2}[7]$.

The forms of both spectra are practically identical, but the value of the photoinduced LD is nearly 5 times smaller than that of the magnetic one.

The most noticeable changes of the magnetic LD at different orientations of the inducing magnetic field (Fig. 1) were observed in the region of the spectrum at wavelengths higher than $600 \mathrm{~nm}$. The absorption spectra investigations [11] showed the presence of $\mathrm{Co}$ of both valences, $\mathrm{Co}^{2+}$ and $\mathrm{Co}^{3+}$, in YIG:Co. The exact assignment of the experimentally observed spectral features to certain transitions in Co ions with a definite valence and in either a tetrahedral or octahedral crystallographic site is not an easy task, because in this region of the spectrum the Co bands are rather wide and overlapping $[11,12]$. Besides, the transitions in octahedral Co have an oscillator strength weaker than those for the tetrahedral ones, and in the spectral region under study the transitions in the $\mathrm{Fe}$ ions also contribute to the spectrum of YIG:Co [13]. The most prominent features originating from transitions in tetrahedral $\mathrm{Co}^{2+}$, in octahedral $\mathrm{Co}$ of both valences, and in $\mathrm{Fe}^{3+}$ ions were determined using the results and analysis of Faraday rotation spectral studies in [12] and [13]. For example, in [12] the bands near $590 \mathrm{~nm}$ and $630 \mathrm{~nm}$ have been identified as arising from transitions in tetrahedral $\mathrm{Co}^{2+}$ and the spectral region near $700 \mathrm{~nm}$ as formed by transitions in octahedral $\mathrm{Co}^{2+}$ and $\mathrm{Co}^{3+}$. The analysis of absorption and Faraday rotation spectra of pure YIG crystals and films, performed in [13], as well as magnetic circular dichroism spectra analysis in [14], allowed attribution of the band near $543 \mathrm{~nm}$ to a transition in tetrahedral $\mathrm{Fe}^{3+}$ and the band in the region of $625 \mathrm{~nm}$ to a transition in octahedral $\mathrm{Fe}^{3+}$.

The coincidence of the photoinduced dichroism spectrum with the magnetic dichroism spectrum indicates that photoinduced $\mathrm{LD}$, like its rigid component, is actually magnetic dichroism. The lightinduced linear dichroism arises as a result of reorientation of magnetic moments after a photoinduced change of magnetic anisotropy.

As has already been proposed in many earlier papers [5-7], light polarized along a certain direction acts on the Co centers in the definite crystallographic positions and causes effective electron transfer between Co ions. This process in its turn results in a change of $\mathrm{Co}$ valences and further redistribution of the Co ions with definite valences among crystallographic sites [5-7] involving the centers in a preferred direction. The process of rearrangement of $\mathrm{Co}$ ions then leads to changing magnetic anisotropy in the film. At $T \sim 30 \mathrm{~K}$ the easy axis of the sample is near the [001] crystallographic axis $[9,10]$, whereas under action of the [100]- or [010]-polarized light the easy axis turns into a hard axes. The easy axis now becomes directed close to any of the [100] or [010] crystallographic axes which are slightly nonequivalent in the real film. As a result, after irradiation the magnetic moments of the magnetic sublattices become oriented along a direction close to any of the cubic axes of the [100] type. The same orientation of magnetic moments results from the action of a magnetic field directed along [100]. It looks quite natural to expect the dichroism spectra to be similar in the two cases, and that is proved by the present experimental observations.

The effect of light with a definite polarization may be therefore presented as the action of an effective magnetic field, with a definite direction, on the YIG:Co film. 
It should be noted that the proposed mechanisms of induced anisotropy could be considered as rather local processes. It is shown that the photoinduced linear dichroism is five times less than that induced by magnetic field, and this fact may be due to the reason that action of the light could be non-uniform, and its effect will be determined by the same factors that make the [100] and [010] directions nonequivalent in the film. Inhomogeneously distributed Co ions as well as impurities, deformations, and elastic stresses may manifest themselves in such a way that in different regions of the sample the easiest axes could take the direction [100] or [010]. This will result in a nonuniform orientation of magnetic moments in the irradiated film and in the formation of small domains in which the preferred dichroism axes will be oriented perpendicularly. This fact will result in the effective compensation of the dichroism, which in the experiment is to be measured in a region much larger than the characteristic size of the inhomogeneities. In the case of the action of a magnetic field, the magnetic moments are oriented rather uniformly, if the magnetic field is of a sufficiently high value.

\section{Conclusion}

Spectra of magnetic linear dichroism sensitive to the orientation of the external magnetic field has been observed in a YIG:Co film. The form of the magnetic LD spectrum for an orientation of the magnetic field along the [100] axis turned out to be similar to the LD spectrum induced by linearly polarized light with polarization along the [100] axis. This similarity of the induced LD spectra leads to the conclusion that the so-called «rigid» compo- nent of the photoinduced optical anisotropy in YIG:Co films is of a magnetic origin.

1. S. Geller, G. Balestrino, A. K. Ray, and A. Tucciarone, Phys. Rev. B27, 326 (1983).

2. A. Maziewski, J. Magn. Magn. Mater. 88, 325 (1990).

3. A. B. Chizhik, S. N. Lyakhiments, A. Maziewski, and M. Tekielak, J. Magn. Magn. Mater. 140-144, 2111 (1995).

4. E. F. Kovalenko and E. L Nagaev, Usp. Fiz. Nauk 148, 561 (1986).

5. A. B. Chizhik, I. I. Davidenko, A. Maziewski, and A. Stupakiewicz, Phys. Rev. B57, 14366 (1998).

6. A. Stupakiewicz, A. Maziewski, I. I. Davidenko, and V. Zablotskii, Phys. Rev. B64, 64405 (2001).

7. A. A.Milner, N. F. Kharchenko, A. Maziewski, and J. M. Desvignes, J. Magn. Magn. Mater. 140-144, 2113 (1995).

8. J. F. Dillon, Jr., E. M. Gyorgy, and J. P. Remeika, Phys. Rev. Lett. 22, 643 (1969).

9. M. Tekielak, W. Andra, A. Maziewski, and J. Taubert, J. Phys. IV 7C, 461 (1997).

10. M. Tekielak, A. Stupakiewicz, A. Maziewski, and J. M. Desvignes, to be published in J. Magn. Magn. Mater. (2002).

11. D. L. Wood and J. P. Remeika, J. Chem. Phys. 46, 3595 (1967).

12. Z. Šimša, J. Šimšova, P. Görnert, and A. Maziewski, Acta Phys. Pol. A76, 277 (1989).

13. W. Wettling, B. Andlauer, P. Koidl, J. Schneider, and W. Tolksdorf, Phys. Status Solidi B59, 63 (1973).

14. R. V. Pisarev, B. Antonini, P. Paroli, and A. J. Tucciarone, J. Magn. Magn. Mater. 54-57, 1391 (1986). 